\title{
Effect of heat processing on thermal stability and antioxidant activity of six flavonoids
}

\author{
Hind Chaaban $^{1}$ | Irina Ioannou ${ }^{1}$ (i) | Latifa Chebil $^{1}$ | Manel Slimane ${ }^{1}$ | \\ Christine Gérardin $^{2}$ | Cédric Paris ${ }^{3}$ | Céline Charbonnel ${ }^{1}$ | Leila Chekir ${ }^{4}$ | \\ Mohamed Ghoul ${ }^{1}$
}

${ }^{1}$ Laboratory of Reactions and Process Engineering (LRGP), Lorraine University, 2 avenue de la Forêt de Haye-TSA, Vandoeuvre Cedex 40602 54518, France

${ }^{2}$ Laboratory of Studies and Research on Wood Material (LERMAB), Lorraine University, Boulevard des Aiguillettes-BP, Vandœuure lès Nancy Cedex 7023954506 , France

${ }^{3}$ Laboratory of Biomolecule Engineering (LIBio), Lorraine University, 2 avenue de la Forêt de Haye-TSA, Vandoeuvre Cedex 40602 54518, France

${ }^{4}$ Laboratory of Cellular and Molecular Biology, Faculty of Dental Medicine, University of Monastir, Rue Avicenne, Monastir 5000, Tunisia

\section{Correspondence}

Irina loannou, Laboratory of Reactions and Process Engineering (LRGP), Lorraine University, 2 avenue de la Forêt de HayeTSA, Vandoeuvre Cedex 4060254518 , France.

Email: irina.ioannou@univ-loraine.fr

\begin{abstract}
The objective of this paper is on the one hand to study the effect of heat processing (30 to $130^{\circ} \mathrm{C}$ for $2 \mathrm{~h}$ ) on the stability and antioxidant activity of six flavonoids (rutin, naringin, eriodictyol, mesquitol, luteolin, and luteolin 7-O glucoside), and on the other hand to establish the relation structure-activity-stability of these compounds. The dependency on temperature of the six kinetics was well described by the Arrhenius law and the main parameters provided by this model are determined and compared in this paper. Activation energies found were $107.3 \mathrm{~kJ} / \mathrm{mol}$ for rutin, 100.6 for naringin, 33.3 for mesquitol, 68.2 for eriodictyol, 51.4 for luteolin, and 120 for luteolin 7-O-glucoside. The data collected showed that glycosylated flavonoids are more resistant than aglycon flavonoids to heat treatment. Moreover, it was also observed that despite the total degradation of some flavonoids, the treated solutions still have an antioxidant activity.

\section{Practical application}

Flavonoids are mainly consumed in processed foods. Thus, flavonoids often undergo heat processing. Knowing the thermal stability and the evolution of their antioxidant activity are particularly relevant in the field of food processing. The results of this study allow information to be collected on the relationship between heat stability-flavonoid structure and antioxidant activity. This information will be useful for the formulation of new food products or for the design of new food processes.
\end{abstract}

\section{KEYWORDS}

antioxidant activity, flavonoid, heat processing, modelling, thermal stability

\section{1 | INTRODUCTION}

Over the last few decades, epidemiological studies have shown that consuming fruit and vegetables rich in phenolic compounds leads to a general well-being of consumers (Tomás-Barberán, Ferreres, \& Gil, 2000). These benefits have been attributed to phenolic compound properties, particularly antioxidant activity which plays a role in this health-promoting capacity (Mellor \& Naumovski, 2016). Among phenolic compounds, flavonoids are the most studied class and much interest is focused on them. The antioxidant activity of flavonoids depends on their structure (Heim, Tagliaferro, \& Bobilya, 2002; Rice-Evans, Miller, $\&$ Paganga, 1996). Modifications of the structure such as alkylation or glycosylation induce a change of their properties; antioxidant activity can be increased or decreased depending on these modifications. Moreover, the human diet is based mainly on the consumption of products having undergone heat treatment, as a transformation step from raw materials to finished products. These processes may have an impact on flavonoid structure and consequently on their antioxidant activities (Bordiga, Travaglia, Mazza, \& Arlorio, 2015; Nayak, Liu, \& Tang, 2015; Saikia, Mahnot, \& Mahanta, 2016).

Flavonoids are classified into nine groups. Compounds belonging to the same group differ from each other in their structure (degree and position of hydroxylation, presence of a substituent, etc.). These differences in structure can affect their stability to heat processing. Most available data concern only two groups-anthocyanins and isoflavones. Studies on anthocyanins have focused on the modeling of flavonoid 
degradation (Patras, Brunton, O'Donnell, \& Tiwari, 2010) while studies on isoflavones have investigated the thermal stability of several isoflavones according to their structure (Stintzing, Hoffmann, \& Carle, 2006; Ungar, Osundahunsi, \& Shimoni, 2003). Apart from these two main studies, Buchner, Krumbein, Rhon, and Kroh (2006) carried out preliminary investigations on quercetin and rutin, and suggested the presence of correlations between structure, antioxidant activity, and heat treatment. Few studies have dealt with the identification of new formed products from flavonoid degradation. This is because they are unstable and lead to further degradations (Dall'acqua, Miolo, Innocenti, \& Cafieri, 2012; Ramešová, Sokolová, Terábek, \& Degano, 2013). Only Buchner et al. (2006) proposed a degradation pathway for quercetin during a thermal process at $100^{\circ} \mathrm{C}$ under oxygen conditions. Thus, a lot still has to be discovered about the impact of heat treatment on the relationship between structure and antioxidant activity of flavonoids and about the identification of the degradation products. Such findings are essential for the development of a tool to predict the effect of heating on flavonoid structure, antioxidant activity, and biological activities. In this paper, the thermal stability of rutin, mesquitol, naringin, eriodictyol, luteolin, and luteolin 7-O glucoside was investigated. These compounds were heated to different temperatures for $2 \mathrm{hr}$. The kinetics of their degradations were modeled and the relationship between the various phytochemical structures and their antioxidant activities were studied.

\section{2 | MATERIALS AND METHODS}

\section{$2.1 \mid$ Chemicals}

Naringin (purity $>95 \%$ ), rutin (purity $>95 \%$ ), 2'-azinobis (3-ethyl-benzothiazoline-6-sulfonic acid) diammonium salt (ABTS) were purchased from Sigma-Aldrich Chemical. Eriodictyol, luteolin, luteolin-7-Oglucoside were purchased from Extrasynthese (purity $>98 \%$ ). Mesquitol (purity $>90 \%$ ) was extracted from a Kenya tree Proposis juliflora (Sirmah, Dumarcay, Masson, \& Gerardin, 2009). Trolox and potassium persulfate were purchased from Fluka. Methanol and ethanol were from Carlo Erba and VWR, respectively. All reagents and solvents were of analytical grade.

\section{2 | Structural differences between flavonoids}

The flavonoids chosen for this study are characterized by a difference in their structure and have a good solubility in water. The flavonoids are subdivided into nine classes. All compounds of the same class have a common structure. Four classes are relevant to study because their common structures differ from each other by one structural element. To study the effect of heat treatment, six molecules belonging to these four classes of flavonoid were selected: flavones (luteolin, luteolin 7-O glucoside) differ from flavonols (rutin) by the absence of the catechol structure. Flavanones (naringin, eriodictyol) differ from flavonols (rutin) by the absence of the enone structure on the C-ring. Flavanols (mesquitol) differ from flavonols (rutin) by the absence of the enone structure and a group carbonyl on the C-ring. In Figure 1, we can see the structure of the six flavonoids. In this study, water was chosen as a food matrix.

\section{3 | Model solutions}

Aqueous solutions of six flavonoids were prepared according to their solubility limit (naringin $0.29 \mathrm{mM}$, rutin $0.041 \mathrm{mM}$, mesquitol $0.93 \mathrm{mM}$, eriodictyol $0.059 \mathrm{mM}$, luteolin $0.0087 \mathrm{mM}$, and luteolin-7-O-glucoside $0.014 \mathrm{mM}$ ). Flavonoids were solubilized in the dark at $25^{\circ} \mathrm{C}$ until maximum solubilization was achieved. The $\mathrm{pH}$ value of the different solutions is equal to $6.6 \pm 0.1$.

\section{4 | Thermal treatment of model solutions}

Thermal treatments were conducted in an oil-based bath (Hubert, W8518D). The different model solutions were filtered through a syringe filter $(0.20 \mu \mathrm{m})$ then $10 \mathrm{~mL}$ samples of each solution were put into screw-cap pyrex tubes and heated from 70 to $130^{\circ} \mathrm{C}$ for a total duration of $120 \mathrm{~min}$. Samples were taken every $15 \mathrm{~min}$ and cooled in an ice bath to $30^{\circ} \mathrm{C}$. Preliminary studies showed that differences in the degradation of flavonoids after $2 \mathrm{hr}$ of heating are not significant. The residual flavonoid content and the antioxidant activity of the solution were measured.

\section{5 | HPLC/DAD analysis}

Flavonoid contents were analyzed in an Elite Lachrom HPLC system (VWR HITACHI) which consisted of a quaternary pump (L-2130), an autosampler (L-2200), and a diode-array detector (L-2455). The analyses were carried put on a C18 $(150 \times 4.6 \mathrm{~mm})$ column (Grace). The different compounds were separated using a water $(A)+$ methanol $(B)$ elution system. Samples were eluted at a flow rate of $1 \mathrm{~mL} / \mathrm{min}$ with a gradient of $95 \%$ water $(\mathrm{A})$ at time 0 up to $100 \%(\mathrm{~B})$ at $10 \mathrm{~min}$ and finally $95 \%(\mathrm{~A})$ at $20 \mathrm{~min}$ before the end of the program. The total duration was $30 \mathrm{~min}$. The injection volume was of $50 \mu \mathrm{L}$ and the column temperature was set at $50^{\circ} \mathrm{C}$. The detection was performed simultaneously at 200, 280, 254, and $350 \mathrm{~nm}$. Flavonoid content was expressed as a percentage compared to the initial content. Then the degradation kinetic was plotted according to heating time. Triplicate measurements were taken for all samples.

\subsection{Identification of the new formed products from flavonoid degradation}

A solution of quercetin at $1 \mathrm{mM}$ was prepared in $100 \mathrm{mM} \mathrm{KCl}$ and $0.36 \mathrm{mM} \mathrm{KOH}$ in order to increase solubility. Aqueous solutions of rutin and eriodictyol were prepared at 0.65 and $0.059 \mathrm{mM}$, respectively. These three solutions were filtered through a syringe filter $(0.20$ $\mu \mathrm{m})$ and heated to $130^{\circ} \mathrm{C}$ for $2 \mathrm{hr}$. Qualitative and semiquantitative analyse of new formed products were realized using an HPLC-MS system (ThermoFisher Scientific, San Jose, CA, USA) consisting of a binary solvent delivery pump connected to a photodiode array detector (PDA) and an LTQ mass spectrometer equipped with an atmospheric pressure ionization interface operating in electrospray negative mode $\left(\mathrm{ESI}^{-}{ }^{-}\right) .25$ 


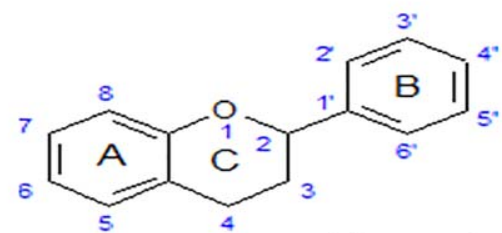

Common structure of flavonoids

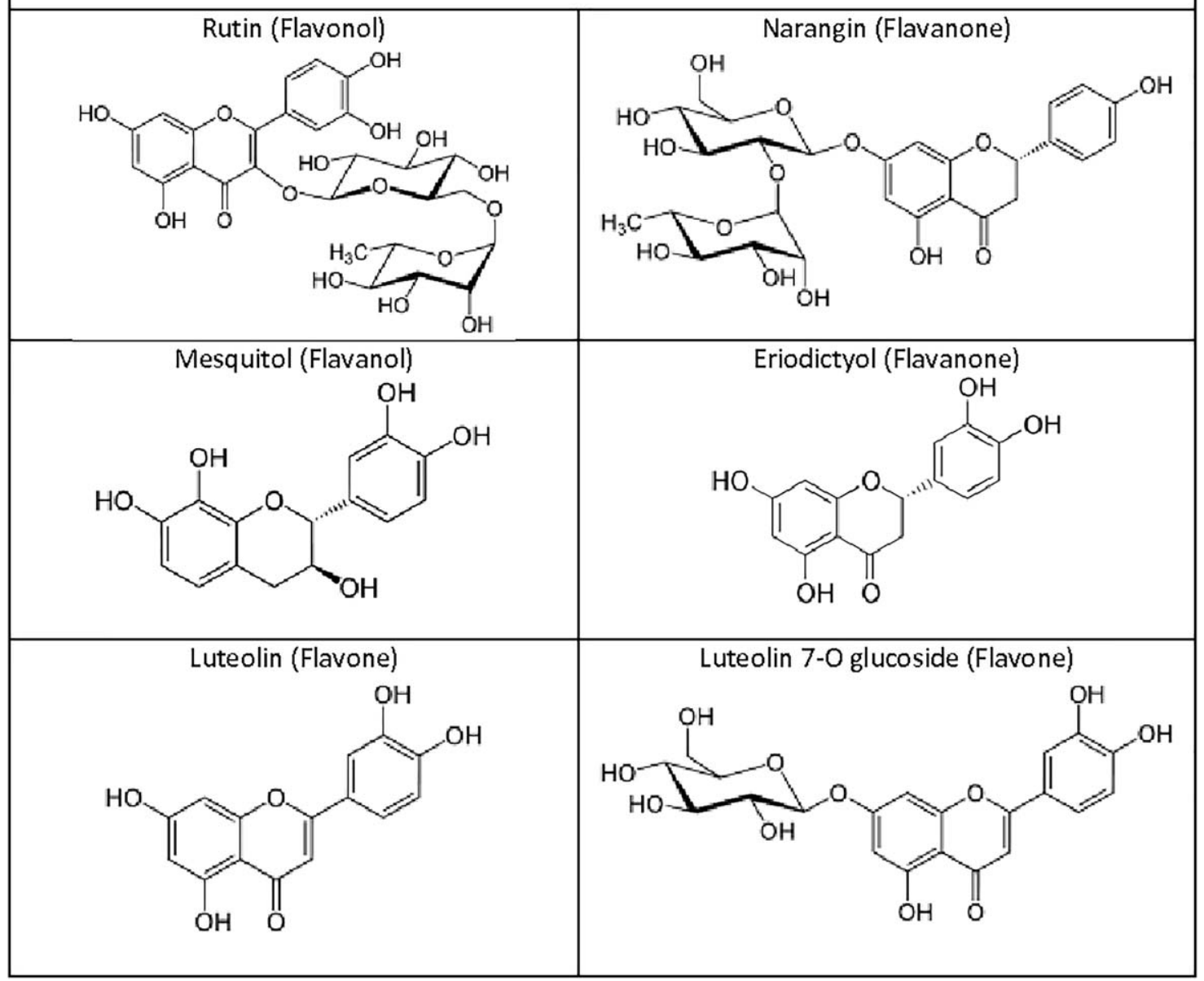

FIGURE 1 Chemical structures of the flavonoid studied

$\mu \mathrm{L}$ of flavonoid degraded solutions was separated on a C18 Alltima (150 mm $\times 2.1 \mathrm{~mm}$ ) column (Grace/Alltech). The flow rate was set at $200 \mu \mathrm{L} \min ^{-1}$. The mobile phases for quercetin and rutin were water modified with trifluoroacetic acid (0.1\%) for A and acetonitrile modified with trifluoroacetic acid (0.1\%) for B. The mobile phase for eriodictyol was water for $\mathrm{A}$ and methanol for $\mathrm{B}$.

Degradation products were eluted using a first isocratic step at $15 \%$ of B for 3 min and then a linear gradient from $15 \%$ to $70 \%$ of B for $21 \mathrm{~min}$. Mass spectrometric conditions were as follows for $\mathrm{ESI}^{-}$ mode: spray voltage was set at $5 \mathrm{kV}$; source gases were set (in arbitrary units $\min ^{-1}$ ) for sheath gas, auxiliary gas, and sweep gas at 40 , 10 , and 10 , respectively; capillary temperature was set at $300^{\circ} \mathrm{C}$; capillary voltage at $-36 \mathrm{~V}$; tube lens, split lens, and front lens voltages at $-80,44$, and $3.25 \mathrm{~V}$, respectively. Ion optic parameters were optimized by automatic tuning using a standard solution of rutin at $0.1 \mathrm{~g} \mathrm{~L}^{-1}$ infused in the mobile phase (A/B: 50/50) at a flow rate of $5 \mu \mathrm{L} \mathrm{min}{ }^{-1}$. Full scan MS spectra (100 to 2,000 m/z) and data dependent $\mathrm{MS}^{2}$ scans for structural investigation were performed using an LTQ (Linear Trap Quadripole). Raw data were processed using the XCALIBUR software program (version 2.1, http://www. thermoscientific.com). $\mathrm{MS}^{2}$ fragmentation data were compared when possible to available literature in order to identify the nature of degradation products.

\section{7 | Antioxidant assay (ABTS)}

Antioxidant activity was determined every 15 min during the thermal treatment by the trolox equivalent antioxidant capacity method. ABTS assays were performed using a spectrofluorimeter SAFAS (Xenius) equipped with a 96-well polystyrene plate. 


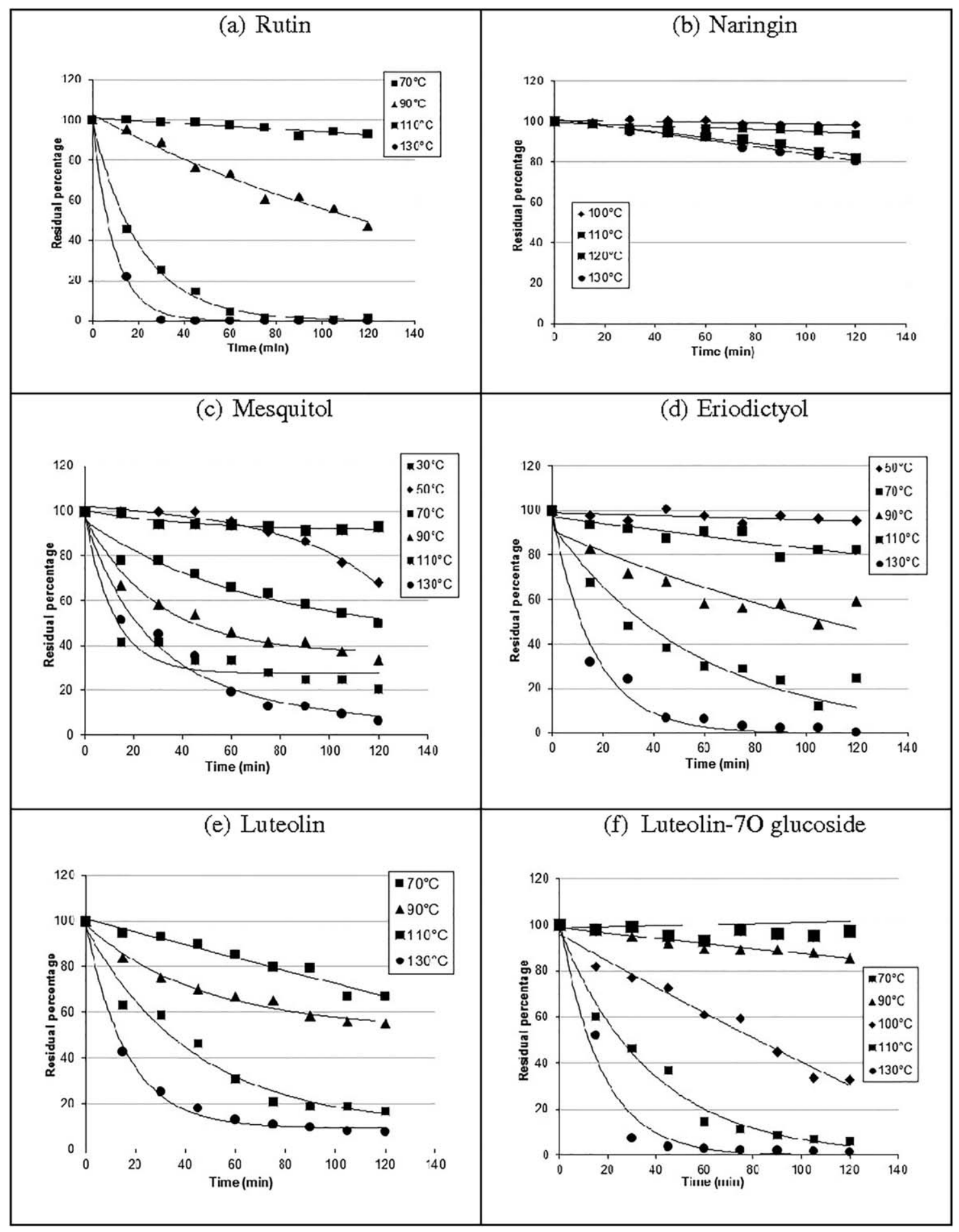

FIGURE 2 Degradation kinetics of flavonoids at different temperatures over $2 \mathrm{hr}$ standard deviations are equal to $2 \%$

Scavenging free radical potentials were tested in an aqueous solution of $\mathrm{ABTS}^{\mathbf{+}}$ according to the method described by Re, Pellegrini, Proteggente, Yang, and Rice-Evans (1999) with modifications for use in microplates. The percentage of inhibition was calculated by the equation: $(I P \%)=100 \times\left(A_{C}-A_{S}\right) / A_{C}$, where $A_{C}$ and $A_{S}$ are the absorbance of the control and test sample, respectively.

Results were expressed as millimoles of TEAC values (Trolox Equivalent Antioxidant Capacity). From this measurement, we deter- mined $\triangle T E A C$. It corresponds to the difference between the total TEAC of the solution and the TEAC due to the residual flavonoid in solution. Triplicate measurements were taken for all samples.

\subsection{Modeling of the degradation kinetics}

To estimate the kinetic parameters of the flavonoid degradation during heat treatment, the order of the reaction was determined. The residual 


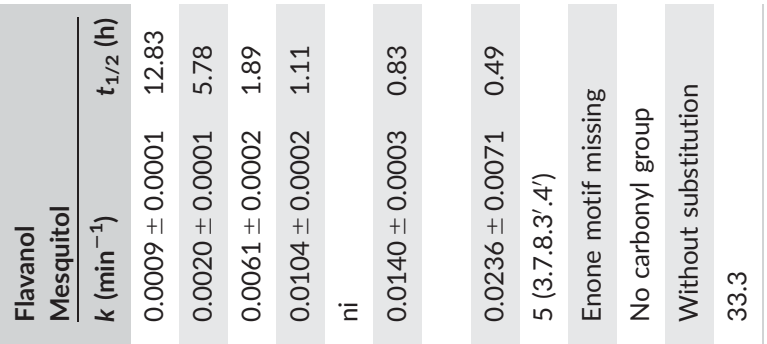
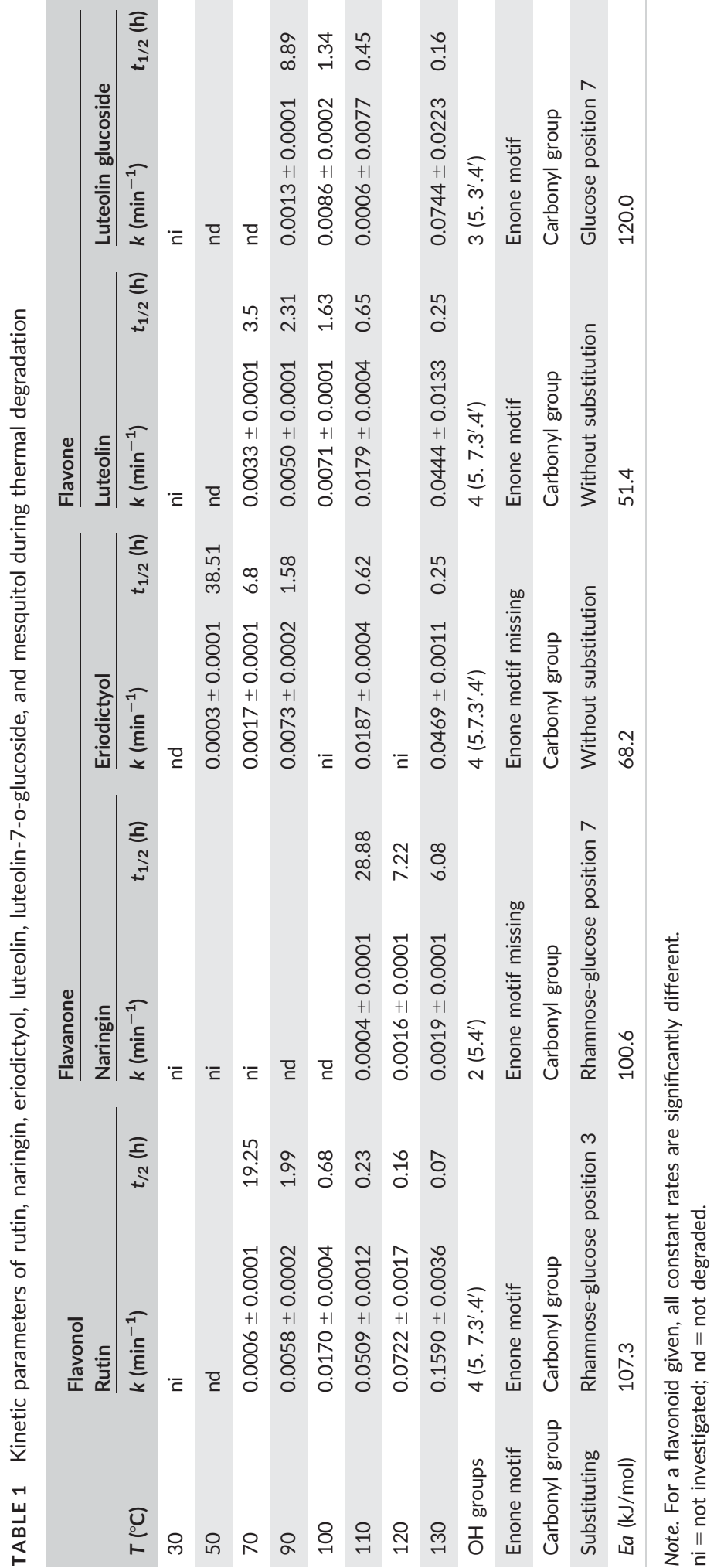
TABLE 2 Antioxidant activity and information on the structure of the different flavonoids

\begin{tabular}{|c|c|c|c|c|c|c|}
\hline & Rutin & Naringin & Eriodictyol & Luteolin & Luteolin glucoside & Mesquitol \\
\hline $\begin{array}{l}\text { Hydroxyl groups } \\
\text { (position. number) }\end{array}$ & $4\left(5.7 .3^{\prime} .4^{\prime}\right)$ & $2\left(5.4^{\prime}\right)$ & $4\left(5.7 .3^{\prime} .4^{\prime}\right)$ & $4\left(5.7 .3^{\prime} .4^{\prime}\right)$ & $3\left(5.3^{\prime} .4^{\prime}\right)$ & $5\left(3.7 .8 .3^{\prime} .4^{\prime}\right)$ \\
\hline Enone motif & $\mathrm{Y}$ & $\mathrm{N}$ & $\mathrm{N}$ & $\mathrm{Y}$ & Y & $\mathrm{N}$ \\
\hline Carbonyl group & 1 & 1 & 1 & 1 & 1 & 0 \\
\hline Substituting & $\begin{array}{l}\text { Rhamnose-glucose } \\
\text { position } 3\end{array}$ & $\begin{array}{l}\text { Rhamnose-glucose } \\
\text { position } 7\end{array}$ & No substituting & No substituting & Glucose position 7 & No substituting \\
\hline $\begin{array}{l}\text { Antioxidant activity } \\
\text { (mmol/l) }\end{array}$ & $3.15^{a} \pm 0.35$ & $0.17^{b} \pm 0.03$ & $1.22^{c} \pm 0.06$ & $5.56^{d} \pm 1.45$ & $4.21^{d} \pm 0.56$ & $0.92^{e} \pm 0.01$ \\
\hline
\end{tabular}

Note. Groups found by Tukey analysis are indicated by letters on the antioxidant activity.

concentration was plotted over time according to (1). The constant rate $k$ corresponds to the slope of the straight line obtained.

$$
\ln \left(C_{t} / C_{0}\right)=-k t
$$

where $C_{0}$ is the initial flavonoid content $(\mathrm{mg} / \mathrm{l})$ and $C_{t}$ is the flavonoid content $(\mathrm{mg} / \mathrm{l})$ after $t$ minutes of heating at a given temperature, $k$ is the reaction rate constant $\left(\mathrm{min}^{-1}\right)$. Half life time $t_{1 / 2}$ can be determined by (2). It corresponds to the heating time required to degrade $50 \%$ of the flavonoid content (min).

$$
t_{1 / 2}=\ln 2 / k
$$

The increase and decrease of temperature during heating were very rapid so we can ignore them and consider that we are in isothermal conditions. Thus, the temperature dependence of the degradation rate constants can be modeled using the Arrhenius law both for temperatures below and above $100^{\circ} \mathrm{C}$. Activation energies $\mathrm{Ea}(\mathrm{kJ} / \mathrm{mol})$ were calculated by the following equation:

$$
\ln k=\ln k_{O}-(E a / R T)
$$

with $R$ is the ideal gas constant $\left(8.314 \mathrm{~J} \mathrm{~K}^{-1} \mathrm{~mol}^{-1}\right)$ and $T$ is the heating temperature $(\mathrm{K})$.

\section{9 | Statistical analysis}

Each experiment was performed using three replicates. The standard deviations were respectively equal to $2 \%$ and $2 \mathrm{mmol}$ trolox/L for residual concentration and $\triangle T E A C$. So, the kinetics of the flavonoid degradation and the antioxidant activity determination are repeatable. Moreover, an ANOVA followed by a Tukey test (freeware $R^{\odot}$ (2.11.1)) was performed to evaluate significant differences on kinetic parameters (k).

\section{3 | RESULTS}

\section{1 | Degradation kinetics of the flavonoids}

The degradation kinetics of rutin, naringin, mesquitol, eriodictyol, luteolin, luteolin-7-O-glucoside in the aqueous system at different temperatures over $2 \mathrm{hr}$ were investigated. The results obtained are summarized in Figure 2. The degradation of rutin (Figure 2a) according to the heating temperature and time showed that, at $70^{\circ} \mathrm{C}$, less than $10 \%$ of rutin was depleted after $2 \mathrm{hr}$ of heating while only $50 \%$ of the flavonoid content was lost at a temperature of $90^{\circ} \mathrm{C}$. For temperatures above $100^{\circ} \mathrm{C}$, a sharp degradation was observed. Thus, after $45 \mathrm{~min}$ at $130^{\circ} \mathrm{C}$, rutin was no longer detectable by the HPLC/DAD system. As indicated in Figure $2 b$, naringin shows a higher stability than rutin when heated to $100,110,120$, and $130^{\circ} \mathrm{C}$. No degradation was observed for temperatures lower than $100^{\circ} \mathrm{C}$ and less than $2 \%$ at $100^{\circ} \mathrm{C}$. At a temperature of $130^{\circ} \mathrm{C}$, only $20 \%$ of naringin content was depleted. Mesquitol was highly sensitive even at low temperatures (Figure 2c). In fact, at $30^{\circ} \mathrm{C}$ about $10 \%$ of reduction was already observed and more than $50 \%$ at $70^{\circ} \mathrm{C}$. At a higher temperature $\left(130^{\circ} \mathrm{C}\right)$ less than $7 \%$ of mesquitol content remained in the solution. The degradation kinetic of eriodictyol is presented in Figure 2d. Little degradation-less than 5\%-was observed for temperatures below $70^{\circ} \mathrm{C}$. For higher temperatures 90 and $110^{\circ} \mathrm{C}, 40 \%$ and $25 \%$ of reduction were observed respectively, whereas, at $130^{\circ} \mathrm{C}$, the residual concentration of eriodictyol was not detectable. Figure $2 \mathrm{e}$ shows the luteolin degradation kinetics. It appears that the luteolin content decreased by $30 \%$ at a temperature of $70^{\circ} \mathrm{C}$. At a temperature of $90^{\circ} \mathrm{C}$, the loss of luteolin was more than $45 \%$. Heating to a temperature of $110^{\circ} \mathrm{C}$ led to a decrease in the luteolin content by more than $80 \%$ and more than $90 \%$ at $130^{\circ} \mathrm{C}$. Luteolin7-O-glucoside showed a higher stability than luteolin (Figure $2 \mathrm{f}$ ) when the heat treatment applied was less than $100^{\circ} \mathrm{C}$. In fact at 70 and $90^{\circ} \mathrm{C}$, the decline in the concentration of luteolin $7-0$-glucoside was $10 \%$ at $70^{\circ} \mathrm{C}$ and $15 \%$ at $90^{\circ} \mathrm{C}$ compared with $30 \%$ and $45 \%$ for luteolin, respectively. At higher temperatures, luteolin-7-O-glucoside became more sensitive than luteolin.

All the flavonoids studied were found to be heat sensitive. Flavonoid degradation increases with heating magnitude and duration. The best curve fit was obtained using an exponential decay. Differences were found between the six flavonoids concerning their thermal stability. These results are in agreement with previous studies which reported a different thermal stability according to the flavonoid structure (Buchner et al., 2006; Stintzing et al., 2006; Ungar et al., 2003). Studies dealing with the modeling of degradation kinetics show either exponential or sigmoidal degradation depending on the kind of flavonoid, the $\mathrm{pH}$, or the temperature (Stintzing et al., 2006; Ungar et al., 2003). 


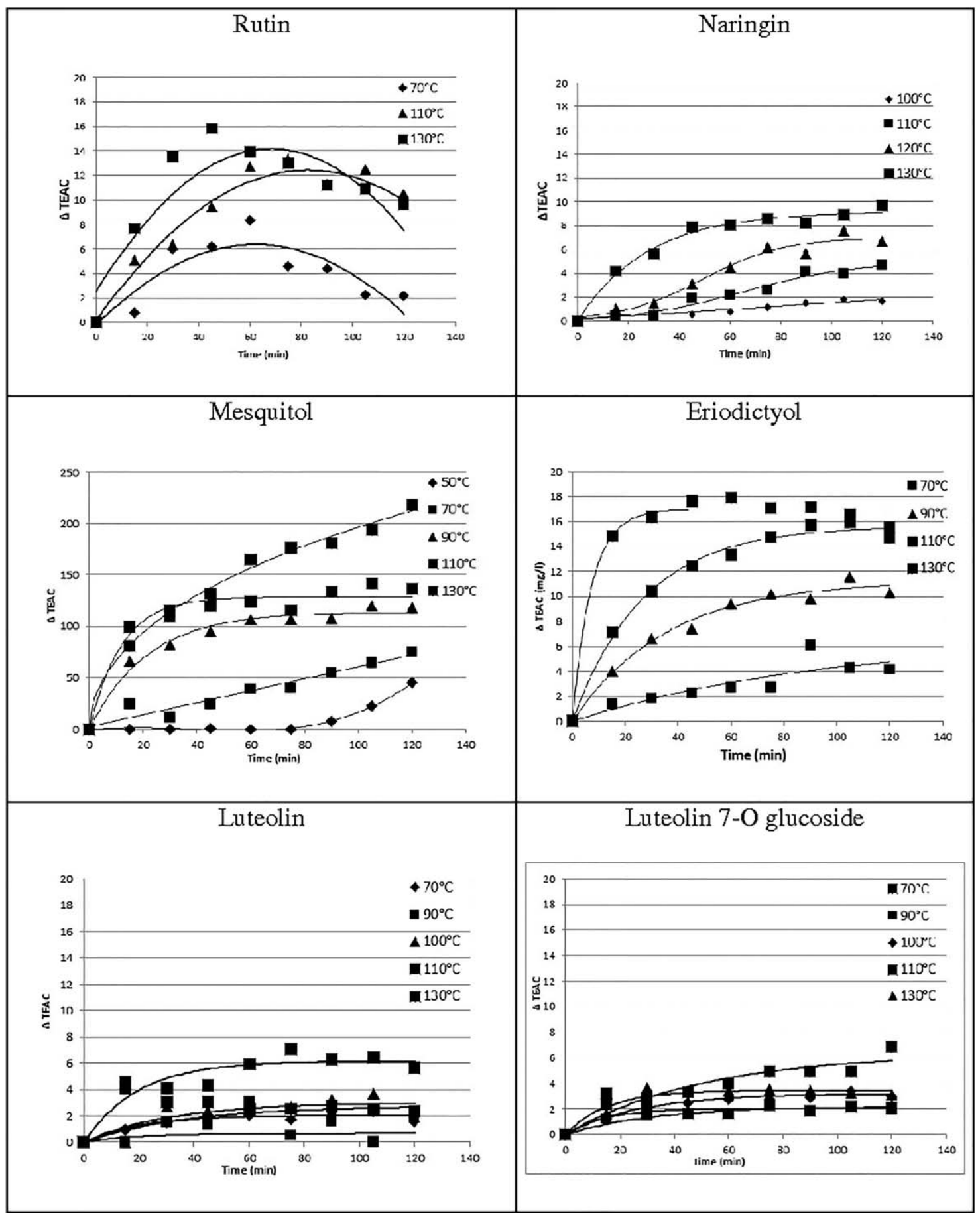

FIGURE 3 Evolution of $\triangle$ TEAC ( $\mathrm{mmol}$ trolox/l) during the thermal treatment standard deviations are equal to $2 \mathrm{mmol}$ trolox/l

\section{2 | Kinetic parameters determination}

To estimate the kinetic parameters of flavonoid degradation during heat treatment, the order of the reaction was determined. Logarithm $(\mathrm{C} / \mathrm{Co})$ was plotted versus heating time. Straight lines were obtained with good determination coefficients (Annex materials). Thus, degradations follow a first-order kinetic. Most studies on the flavonoid degradation have reported that this degradation follows first-order kinetic (Patras et al., 2010). Constant rates and half-life times are given in Table 1. The Arrhenius model was used to calculate temperature dependence. The
Arrhenius model is found to be a reliable mathematical method according to the determination coefficient values (Annex materials).

The rate of degradation $(k)$ varies for a given compound according to temperature, and for a given temperature it varies depending on the nature of the compound studied. However, the magnitude of these variations according to temperature is not the same for all studied compounds. As an illustration of this phenomenon, the $k$ values obtained at $130^{\circ} \mathrm{C}$ were compared. It appears that naringin and mesquitol are the flavonoids that are characterized by the slowest kinetics of degradation 
TABLE 3 LC-MS data obtained for the thermal degradation of quercetin, rutin, and eriodictyol

\begin{tabular}{|c|c|c|c|c|}
\hline Peak & Retention time (min) & $m / z$ values $[M-H]^{-}$ & $\mathrm{m} / \mathrm{z}$ values $\mathrm{MS} / \mathrm{MS}$ & Suggested compound \\
\hline \multicolumn{5}{|c|}{ Quercetin $(1 \mathrm{mM})$ in $100 \mathrm{mM} \mathrm{KCl}$ and $0.36 \mathrm{mM} \mathrm{KOH}$} \\
\hline P1 & 9.93 & 317 & $191-299-207-163$ & 2-(3',4'-dihydroxybenzoyl)-2,4,6-trihydroxybenzofuran-3(2H)-one \\
\hline P2 & 11.82 & 349 & 330 & $\left(3^{\prime}, 4^{\prime}, 5^{\prime}, 6^{\prime}\right.$-tetrahydroxybenzoyl)-2,4,6-trihydroxybenzofuran-3(2H)-one \\
\hline P3 & 12.40 & 333 & ND & $\left(3^{\prime}, 4^{\prime}, 5^{\prime}\right.$-trihydroxybenzoyl)-2,4,6-trihydroxybenzofuran-3(2H)-one \\
\hline P4 & 3.28 & 181 & ND & 2-(3,4-dihydroxyphenyl)-2-oxoacetic acid \\
\hline P5 & 4.67 & 544 & ND & Unidentified \\
\hline P6 & 5.46 & 169 & 151 & 2,4,6-trihydroxybenzoic acid \\
\hline P7 & 24.03 & 297 & 183 & Unidentified \\
\hline \multicolumn{5}{|c|}{ Rutin $(0.65 \mathrm{mM})$ in water } \\
\hline P1 & 4.53 & 278 & 197 & Unidentified \\
\hline P2 & 6.52 & 153 & ND & Protocatechuic acid \\
\hline P3 & 8.83 & 1,217 & $909-601$ & Dimer \\
\hline P4 & 12.12 & 463 & 301 & Quercetin-glucose \\
\hline P5 & 13.41 & 607 & 317 & Unidentified \\
\hline P6 & 17.42 & 301 & $179-151$ & Quercetin \\
\hline \multicolumn{5}{|c|}{ Eriodictyol $(0.059 \mathrm{mM})$ in water } \\
\hline P1 & 8.46 & 197 & 133 & 3-(3,4-dihydroxyphenyl)-3-hydroxypropanoic acid \\
\hline P2 & 11.87 & 165 & $137-121$ & 3-(3,4-dihydroxyphenyl) propanal \\
\hline P3 & 13.53 & 301 & $286-242-283-257$ & Unidentified \\
\hline
\end{tabular}

$\mathrm{ND}=$ no data obtained.

with a $\mathrm{k}$ of 0.0019 and $0.0236 \mathrm{~min}^{-1}$, respectively, followed by eriodictyol and luteolin with, respectively, 0.0469 and $0.0444 \mathrm{~min}^{-1}$. Then, the most rapid kinetics were encountered with rutin and luteolin 7-O glucoside with 0.1590 and $0.0744 \mathrm{~min}^{-1}$, respectively. The analysis of the activation energies found by the Arrhenius law shows differences between the six flavonoids. Mesquitol needs $33.3 \mathrm{~kJ} / \mathrm{mol}$ to be degraded, $51.4 \mathrm{~kJ} / \mathrm{mol}$ for luteolin, 68.2 for eriodictyol, 100.6 for naringin, 107.3 for rutin, and 120 for luteolin 7-O glucoside. Activation energy corresponds to the quantity of energy needed to initiate the degradation reactions. The higher the activation energy, the more stable the flavonoid will be at high temperatures. Indeed, the flavonoid which is sensitive to a temperature of $30^{\circ} \mathrm{C}$ (mesquitol) needs only $33.3 \mathrm{~kJ} / \mathrm{mol}$, whereas the flavonoid sensitive to higher temperatures (rutin, luteolin 7-O glucoside) needs more than $100 \mathrm{~kJ} / \mathrm{mol}$. The differences observed between the kinetics of the degradation of the six flavonoids cannot be explained solely by their belonging to a given class of flavonoid. The structure of these molecules also seems to be at the origin of these differences. In fact, Buchner et al. (2006) explained that rutin is less sensitive to heat than quercetin because of the presence of a glycosyl moiety. With a view to elucidating the relation between the structure and heat sensitivity of these compounds, the structural characteristics and their kinetic parameters are summarized in Table 1. This table shows a difference between the glycosylated flavonoids and the aglycones. Indeed, activation energy was higher for the glycosylated: rutin $(107 \mathrm{~kJ} / \mathrm{mol})$, naringin $(100 \mathrm{~kJ} / \mathrm{mol})$, and luteolin $7-0$ glucoside $(120 \mathrm{~kJ} / \mathrm{mol})$. This can be explained by the presence of a substituent, because additional energy is needed to break the osidic bond between the heteroside and the flavonoid (Buchner et al., 2006; Da Costa, Barbosa Filho, Do Nascimento, \& Macedo, 2002; Rohn, Buchner, Driemel, Rauser, \& Kroh, 2007). However, as rutin is more sensitive to heat than naringin, the difference between the two could be explained by the presence of a double bond in the $\mathrm{C}$-ring $\mathrm{C}$ for rutin, and additional energy is needed to break it. For the aglycon flavonoids, mesquitol has low activation energy in comparison to eriodictyol and luteolin. This can be explained by the absence of a carbonyl group for mesquitol, so the energy needed for its degradation will be lower. However, as the change (hydroxylation, glycosylation) in the structure of the six flavonoids takes place in different parts of the molecules, it is rather difficult to attribute the observed behavior to one substitution at a time.

\section{3 | Antioxidant activity evolution}

Before the heat treatment, the antioxidant activity of each flavonoid was characterized. Flavonoids having the highest antioxidant capacity are luteolin, luteolin-7-O-glucoside, and rutin. However, the capacities of the three compounds are different and equal to $5.56 \pm 1.45$, $4.21 \pm 0.56$, and $3.15 \pm 0.35 \mathrm{mmol} / \mathrm{l}$, respectively (Table 2). This behavior can be explained by the presence in these three compounds of the enone structure (2-3 double bond of the C-ring) conjugated with a carbonyl group in $\mathrm{C} 4$, which is missing in the structure of the three other flavonoids. It has been indicated that this structure is responsible for an increase of antioxidant activity (Heim et al., 2002). Among luteolin, luteolin 7-Oglucoside, and rutin, the aglycon flavonoid has a higher TEAC. These results showed that glycosylation or 


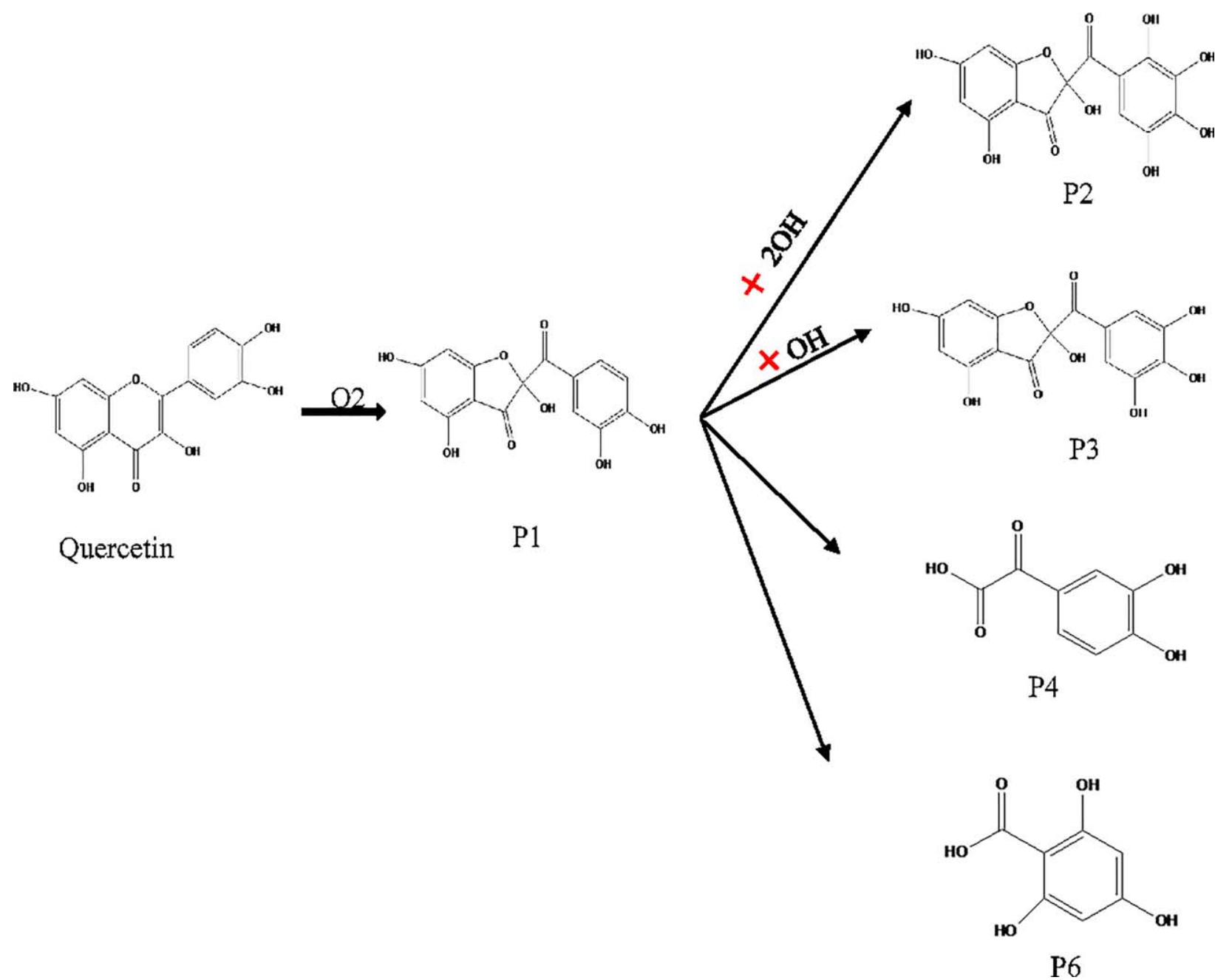

FIGURE 4 Thermal degradation pathway of quercetin

methylation provokes a significant decrease in antioxidant activity and particularly when the substitute is in position 3 (Van Acker et al., 1996). Indeed, the hydroxyl in position 3 confers antioxidant activity to molecules (Heim et al., 2002).

Eriodictyol, mesquitol, and naringin present, respectively, an antioxidant activity of $1.22 \pm 0.06,0.92 \pm 0.01$, and $0.17 \mathrm{mmol} / \mathrm{l} \pm 0.03$. Naringin has a very low antioxidant activity in comparison to eriodictyol and mesquitol because of the glycosylation and the absence of the $3^{\prime}, 4^{\prime}$-catechol structure. It has been reported that the presence of two hydroxyl groups in position $3^{\prime}$ and $4^{\prime}$ on the B-ring increases flavonoid antioxidant activity (Heim et al., 2002). For eriodictyol and mesquitol, the presence of a hydroxyl group in position 3 does not counterbalance the absence of the enone structure. The number of hydroxyl groups seems to be at the origin of the high antioxidant activity of eriodictyol compared to mesquitol. Antioxidant activity of a flavonoid seems to be linked to some structural elements. All factors that change the flavonoid structure lead to an evolution of antioxidant activity. In Figure 3, the $\triangle T E A C$ is given according to heating time at different temperatures. For a given time $\left(t_{i}\right), \triangle T E A C(m m o l$ trolox/l) is defined as the difference between the measured TEAC of the solution containing both residual flavonoid and product of degradation, and the predicted
TEAC, taking into account only the measured residual concentration of the flavonoid studied.

According to the evolution of $\triangle T E A C$ with time, three cases can be observed: (i) an increase of $\triangle T E A C$ shows that the products formed have an antioxidant activity that is higher than the native flavonoid; (ii) a decrease of $\triangle T E A C$ indicates a lower antioxidant activity of the degradation products; and (iii) a $\triangle T E A C$ constant means that degradation products have the same antioxidant activity as the native flavonoid.

For luteolin and luteolin 7-O glucoside, standard deviations range between 1 and 2 , so there is no increase of $\triangle T E A C$ with temperature or heating time. Thus we can conclude that degradation products have an antioxidant activity that is similar to that of native flavonoids.

For the other flavonoids, standard deviations are low in comparison with TEAC values (inferior to 1), so we can state that there is an increase of $\triangle T E A C$ during the heat treatment. We notice that this increase is higher when temperature increases. So rutin, naringin, eriodictyol, and mesquitol lead to degradation products with an antioxidant activity. Similar findings were reported when rutin samples were heated to $100^{\circ} \mathrm{C}$ for $300 \mathrm{~min}$. Despite the small amount of rutin left in the samples after thermal treatment, the solution still possessed antioxidant ability (Buchner et al., 2006). 


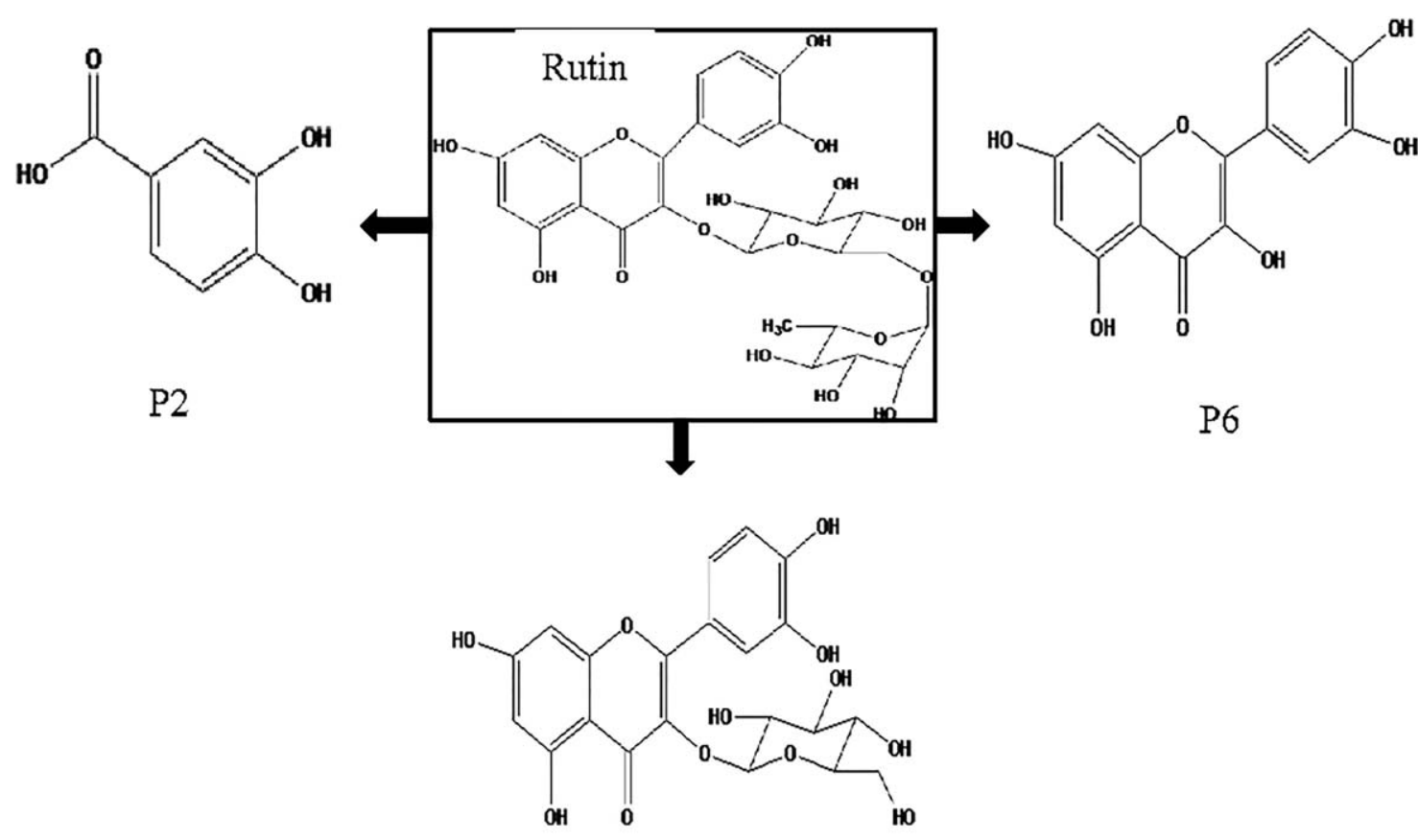

FIGURE 5 Thermal degradation pathway of rutin

Depending on the structure of the molecule and heat treatment behavior, the evolution of the antioxidant activity is different. For mesquitol, an increase of 200 at $130^{\circ} \mathrm{C}$ after $2 \mathrm{hr}$ was observed, whereas for eriodictyol, the increase was only of 17 and 9 for naringin. Moreover, the antioxidant activity of rutin increased during the first hour of treatment then dropped progressively. This can be attributed to a sequential formation and degradation of the degradation compound during heat treatment.

\section{4 | Identification of flavonoid degradation products}

As explained before, the evolution of the antioxidant activity of the six flavonoids is different according to the flavonoid structure and the temperature of the thermal treatment. The few data available in the literature on the new formed products after flavonoid degradation indicated the existence of different degradation pathways leading to different new formed products (Dall'acqua et al., 2012; Ramešová et al., 2013). Thus, the new formed products would be different according to the kind of degradation (temperature, oxygen, light) and the structure of the native flavonoid. Results found on thermal degradation of quercetin (aglycon), rutin (glycosylated), and eriodictyol (aglycon without the enone structure) are in agreement with conclusions from the literature. The quercetin is considered as a reference because it has already been studied in the literature and it constitutes the aglycon part of rutin. To identify the new formed products, samples were taken at the end of the thermal treatment and analyzed by LC-MS.

\subsubsection{Identification of new formed products from quercetin degradation}

The results obtained showed that quercetin is highly sensitive to temperature. The thermal degradation begins at low temperature during the step of solubilization. Products P1 (2-( $3^{\prime}, 4^{\prime}$-dihydroxybenzoyl)-2,4,6trihydroxybenzofuran-3(2H)-one), P2 ((3', $4^{\prime}, 5^{\prime}, 6^{\prime}$-tetrahydroxybenzoyl)2,4,6-trihydroxybenzofuran-3(2H)-one), P3 ((3', $4^{\prime}, 5^{\prime}$-trihydroxybenzoyl)2,4,6-trihydroxybenzofuran-3(2H)-one) were obtained after solubilization at $35^{\circ} \mathrm{C}$. During the thermal treatment $\left(130^{\circ} \mathrm{C}, 2 \mathrm{hr}\right)$, quercetin, $\mathrm{P} 1$, and $\mathrm{P} 2$ are totally degraded and lead to the formation of P4 (2-(3,4-dihydroxyphenyl)-2-oxoacetic acid), P5 (unidentified product), P6 (2,4,6-trihydroxybenzoic acid), and P7(unidentified product). The molar masses of the new formed products are summarized Table 3 and the quercetin degradation pathway is indicated in Figure 4. The chromatograms obtained by LC-MS are given in Annex materials.

A similar methodology was applied for the other two flavonoids.

\subsubsection{Identification of new formed products from rutin degradation}

As for quercetin, the thermal degradation of rutin was assessed by LCMS (chromatograms presented in Annex materials). It appears that after $2 \mathrm{hr}$ of heating, six products were observed. Four molecules were identified: P2 (protocatechuic acid), P3 (dimer of rutin), P4 (quercetin-glucose), and P6 (quercetin). A degradation pathway is presented in Figure 5. For the dimer of rutin, different structures can be found (Anthoni, Chebil, Lionneton, Magdalou, Humeau, \& Ghoul, 2011), they are proposed in Figure 6 . These results indicated that the thermal degradation 


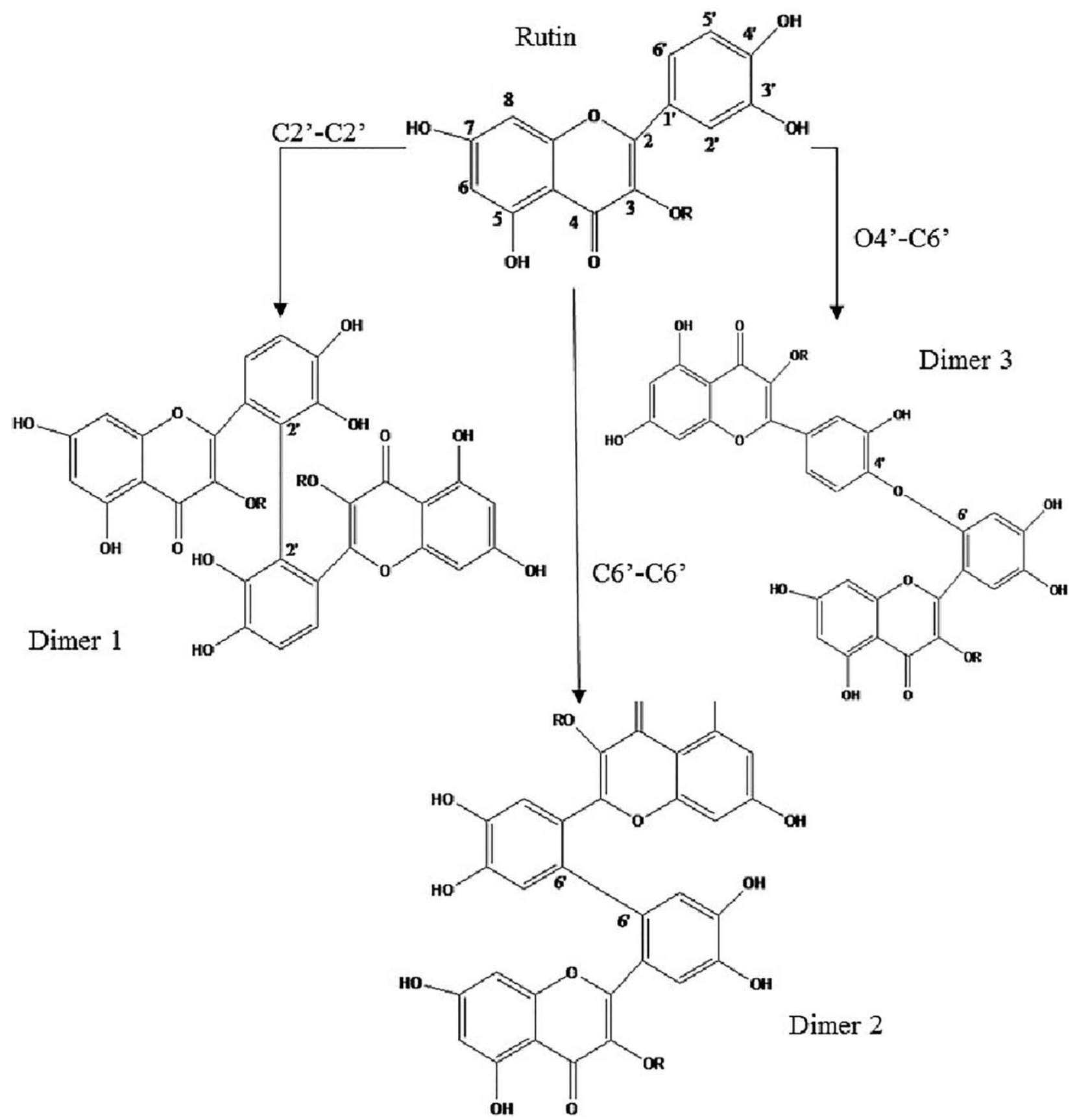

FIGURE 6 Structures of rutin dimer (Anthoni et al., 2011)

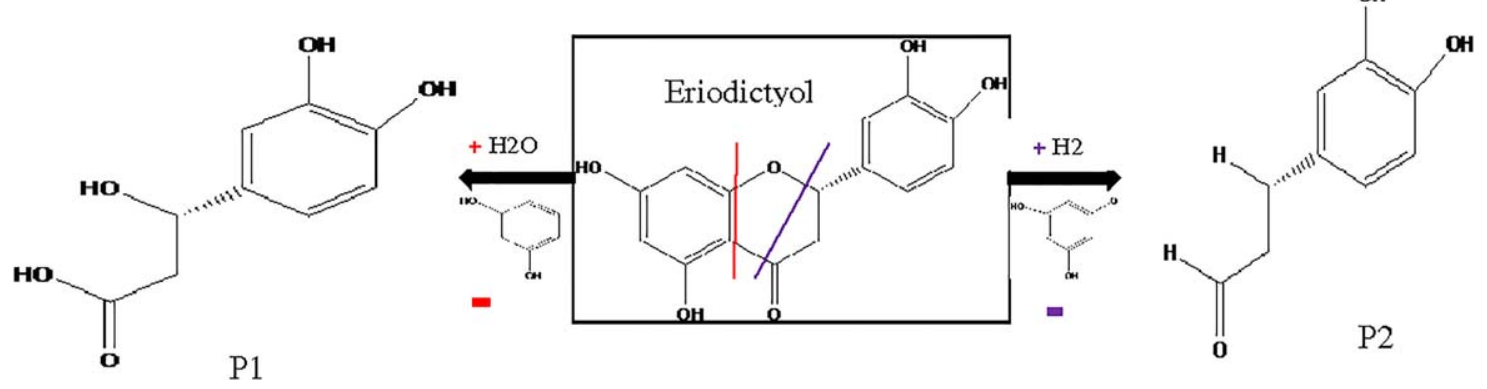

FIGURE 7 Thermal degradation pathway of eriodictyol 
pathway of quercetin and rutin are not similar despite the presence of genin in the two structures.

\subsubsection{Identification of new formed products from eriodictyol degradation}

The chromatograms of the thermal degradation of eriodictyol are indicated in Annex materials. The molar masses of the different byproducts are summarized in Table 3. After $2 \mathrm{hr}$ of thermal treatment, three products P1 (3-(3,4-dihydroxyphenyl)-3-hydroxypropanoic acid), P2 (3-(3,4-dihydroxyphenyl) propanal), and P3(unidentified product) were observed. A thermal degradation pathway is shown in Figure 7.

\section{4 | CONCLUSION}

Temperature has an effect on the stability of flavonoids and their biological activity. According to their structure, flavonoids are more or less sensitive to heat treatment. Glycosylated flavonoids are more resistant to heat treatment than aglycon flavonoids. The degradation depends on structural solidity. Therefore, a double bond needs more energy in order to be degraded. Modifications of structure lead to changes in antioxidant activity. According to the degradation products synthesized: (i) antioxidant activity can decrease, which means that degradation products have a lower antioxidant activity, (ii) antioxidant activity remains constant, indicating that degradation products have the same antioxidant activity as native flavonoids, and (iii) antioxidant activity can increase, so the degradation products have a higher antioxidant activity. Degradation products of three flavonoids were identified and we concluded that the thermal degradation pathways are different according to the structure of the flavonoid. The new formed products exhibit residual antioxidant activity sometimes superior to that of native molecules.

\section{REFERENCES}

Anthoni, J., Chebil, L., Lionneton, F., Magdalou, J., Humeau, C., \& Ghoul, M. (2011). Automated analysis of synthesized oligorutin and oligoesculin by laccase. Canadian Journal of Chemistry, 89, 964-970.

Bordiga, M., Travaglia, F., Mazza, G., \& Arlorio, M. (2015). Evaluation of the effect of processing on cocoa polyphenols: Antiradical activity, anthocyanins and procyanidins profiling from raw beans to chocolate. International Journal of Food Science and Technology, 50, 840-848.

Buchner, N., Krumbein, A., Rhon, S., \& Kroh, L. W. (2006). Effect of thermal processing on the flavonols rutin and quercetin. Rapid Communications in Mass Spectrometry, 20, 3229-3235.

Da Costa, E. M., Barbosa Filho, J. M., Do Nascimento, T. G., \& Macedo, R. O. (2002). Thermal characterization of the quercetin and rutin flavonoids. Thermochim Acta, 392-393, 79-84.

Dall'acqua, S., Miolo, G., Innocenti, G., \& Cafieri, S. (2012). The photodegradation of quercetin: Relation to oxidation. Molecules, 17, 8898-8907.

Heim, K. E., Tagliaferro, A. R., \& Bobilya, D. J. (2002). Flavonoid antioxidants: Chemistry, metabolism and structure-activity relationships. Journal of Nutritional Biochemistry, 13, 572-584.

Mellor, D. D., \& Naumovski, N. (2016). Effect of cocoa in diabetes: The potential of the pancreas and liver as key target organs, more than an antioxidant effect? International Journal of Food Science and Technology, 51, 829-841.

Nayak, B., Liu, R. H., \& Tang, J. (2015). Effect of processing on phenolic antioxidants of fruits, vegetables, and grains-A review. Critical Reviews in Food Science, 55, 887-918.

Patras, A., Brunton, N. P., O'Donnell, C., \& Tiwari, B. K. (2010). Effect of thermal processing on anthocyanin stability in foods: Mechanisms and kinetics of degradation. Trends in Food Science \& Technology, 21, 3-11.

Ramešová, S., Sokolová, R., Terábek, J., \& Degano, I. (2013). The oxidation of luteolin, the natural flavonoid dye. Electrochimica Acta, 110, 646-654.

Re, R., Pellegrini, N., Proteggente, A., Yang, M., \& Rice-Evans, C. (1999). Antioxidant activity applying an improved ABTS radical cation decolorization assay. Free Radical Biology and Medicine, 26, 1231-1237.

Rice-Evans, C. A., Miller, N. J., \& Paganga, G. (1996). Structure-antioxidant activity relationships of flavonoids and phenolic acids. Free Radical Biology and Medicine, 20, 933-956.

Rohn, S., Buchner, N., Driemel, G., Rauser, M., \& Kroh, L. W. (2007). Thermal degradation of onion quercetin glucosides under roasting conditions. Journal of Agricultural and Food Chemistry, 55, 1568-1573.

Saikia, S., Mahnot, N. K., \& Mahanta, N. L. (2016). A comparative study on the effect of conventional thermal pasteurisation, microwave and ultrasound treatments on the antioxidant activity of five fruit juices. Food Science and Technology International, 22, 288-301.

Sirmah, P., Dumarcay, S., Masson, E., \& Gerardin, P. (2009). Unusual amount of (-) mesquitol from the heartwood of Prosopis juliflora. Natural Product Research, 23, 183-189.

Stintzing, F. C., Hoffmann, M., \& Carle, R. (2006). Thermal degradation kinetics of isoflavone aglycones from soy and red clover. Molecular Nutrition \& Food Research, 50, 373-377.

Tomás-Barberán, F. A., Ferreres, F., \& Gil, M. I. (2000). Antioxidant phenolic metabolites from fruit and vegetables and changes during postharvest storage and processing. In Atta-ur-Rahman (Ed.), Studies in natural products chemistry, 23, (pp. 739-795). Elsevier Science, Amsterdam.

Ungar, Y., Osundahunsi, O. F., \& Shimoni, Y. (2003). Thermal stability of genistein and daidzein and its effect on their antioxidant activity. Journal of Agricultural and Food Chemistry, 51, 4394-4399.

Van Acker, S. A. B. E., Van Den Berg, D. J., Tromp, M. L. N. J. L., Griffioen, D. S. E. H., Van Bennekom, W. P., Van Der Vijgh, W. J. F., \& Bast, A. (1996). Structural aspects of antioxidant activity of flavonoids. Free Radical Biology and Medicine, 20, 331-342.

\section{SUPPORTING INFORMATION}

Additional Supporting Information may be found online in the supporting information tab for this article.

How to cite this article: Chaaban $\mathrm{H}$, loannou I, Chebil L, et al. Effect of heat processing on thermal stability and antioxidant activity of six flavonoids. J. Food Process. Preserv. 2017;00: e13203. https://doi.org/10.1111/jfpp.13203 